\title{
Nuclear Magnetic Resonance Analysis of Glucose Levels in Weanling Piglets Plasma as a Function of Deoxynivalenol Exposure
}

\author{
Richard P. Hopton, ${ }^{1,2}$ Isabelle P. Oswald, ${ }^{3}$ Laura J. Hardie, ${ }^{2}$ \\ Paul C. Turner, ${ }^{2,4}$ and Julie Fisher ${ }^{1}$ \\ ${ }^{1}$ School of Chemistry, University of Leeds, Leeds LS2 9JT, UK \\ ${ }^{2}$ Molecular Epidemiology Unit, Leeds Institute of Genetics, Health and Therapeutics, University of Leeds, Leeds, UK \\ ${ }^{3}$ INRA, UMR1331, Toxalim, Research Centre in Food Toxicology, F-31027 Toulouse, France \\ ${ }^{4}$ MIAEH, School of Public Health, University of Maryland, College Park, MD, USA
}

Correspondence should be addressed to Julie Fisher, j.fisher@chem.leeds.ac.uk

Received 30 August 2012; Accepted 16 October 2012

Academic Editors: P. Campíns-Falcó, T. Macko, and A. Tsantili-Kakoulidou

Copyright (C) 2012 Richard P. Hopton et al. This is an open access article distributed under the Creative Commons Attribution License, which permits unrestricted use, distribution, and reproduction in any medium, provided the original work is properly cited.

Deoxynivalenol (DON) is a mycotoxin contaminant of cereal crops, and whilst animal toxicity has demonstrated its role at low doses in animals, and in human toxicity in general remains unclear. Here we investigated potential biological markers in sera using samples obtained from a previous DON-controlled feeding study on weanling piglets. Piglets $(n=10$ per group) received diets containing 0 (control), 240, 560-or $840 \mu \mathrm{g}$ DON $/ \mathrm{kg}$ feed for 28 days. Plasma samples collected at the end of the DON exposure trial were reassessed, using nuclear magnetic resonance (NMR). A modest trend was observed between plasma glucose concentration and DON ingestion $(P=0.04)$. Plasma DON $(P=0.04)$ and plasma protein-associated DON $(P=0.08)$ were moderately correlated with DON ingestion in a more limited survey of a subset of samples used for glucose analysis $(n=20)$. This is a preliminary study but suggests that a more detailed survey of the temporal nature of these biological measures is warranted.

\section{Introduction}

Deoxynivalenol (DON) is a member of the trichothecene family of mycotoxins produced by Fusarium graminearum (Gibberella zeae) and F. culmorum fungi $[1,2]$. In temperate regions of the world DON is one of the most frequent mycotoxin contaminants of cereal crops intended for human consumption $[1,2]$. DON is stable for most cooking methods [3] and as a result remains in the food chain [4]. An extensive survey of $>45,000$ cereal samples from a number of European countries revealed that of those tested for DON, $57 \%$ were contaminated, and $7 \%$ had contamination levels exceeding $750 \mu \mathrm{g} / \mathrm{kg}$ [2]. Based on contamination levels of the cereals and mean consumption patterns these authors concluded that in some individuals the recommended tolerable daily intake (rTDI) (1000 ng/kg bw/day) [5] would be exceeded, and that young children as a group were at greatest risk of exceeding the TDI. Epidemiological data suggests that gastroenteritis, growth faltering, and an increased susceptibility to infections are possible consequences of DON exposure [6]. The toxicity of DON has been established in various animal models with significant differences in sensitivity between species [6]. To date, data indicating limited human capacity to detoxify DON to the deepoxy metabolite, DOM-1 [7-11], suggest that humans may be one of the more sensitive species to DON toxicity.

In order to better understand the potential health risks of DON it is important to use validated biomarkers that reflect both exposure and effect. To that end blood and urinary analysis by proteomic and metabolomics approaches are now emerging as important tools [7, 12-14]. Given the strong links between DON exposure, nutrient bioavailability/retention and growth faltering in animals, one interesting target for DON, are the glucose transporter proteins. In vitro 
analysis of the effects of DON on isolated sections of chicken jejunum indicated inhibitory effects on glucose transport $[15,16]$. Glucose transporters are a family of proteins whose activity is involved in a number of actions including glucose uptake from the gut, retention in the kidney, pancreatic signalling for insulin, transport out of the liver, and skeletal muscle uptake. Inhibition of glucose uptake from the gut has been postulated as one potential mechanism of DON initiated animal health problems $[15,16]$. Though at high doses of DON $(2.2-2.5 \mathrm{mg} / \mathrm{kg}$ in feed) in swine a significant increase in plasma glucose has been reported [17], of course these doses will not reflect typical human exposures.

Previously we reported a DON dosing study in swine using DON concentrations in feed at levels more relevant to possible human exposures (a control and three groups280,560 , or $840 \mu \mathrm{g}$ DON $/ \mathrm{kg}$ ) [18]. The effects of dosing were analysed in terms of animal performance, haematological, biochemical, and immunological variables. Animal feed intake and body weight were unaffected by dose. Eighteen biochemical variables were assessed, including glucose, urea, creatinine and cholesterol levels, all apparently independent of DON dose [18] though a modest positive trend was observed between the DON feed level and plasma glucose concentration. Here we initially surveyed the complete plasma metabolic profile using ${ }^{1} \mathrm{H}$ NMR but then focused on the glucose content of these samples in relation to DON feed levels. Whilst DON feed contamination levels were known, no biomeasures of DON exposure were conducted in the earlier study. Consequently assessment of plasma DON levels was additionally conducted using methods modified from urinary DON analysis [19] and was reported with reference to the NMR generated glucose measures.

\section{Materials and Methods}

2.1. Animals. Forty-eight pig plasma samples were previously acquired and stored at $-32^{\circ} \mathrm{C}$ until required. Full study details are available in Accensi et al. [18]. In brief, the pigs were housed individually with free access to feed and water. Diets were such that the DON content was $0,280,560$, and $840 \mu \mathrm{g} / \mathrm{kg}$ of feed per day for four weeks, for groups 1-4, respectively, for each of 12 animals per group. At the end of the experiment, blood samples were aseptically collected from the left jugular vein. Blood was collected in tubes (Vacutainer, Becton-Dickinson, Le pont de Claix, France) containing sodium heparin. Plasma samples, obtained after centrifugation of heparinized blood at $600 \times \mathrm{g}$, were stored at $-20^{\circ} \mathrm{C}$ until analysed.

2.2. Plasma NMR Analysis. The full NMR spectrum, recorded for all samples, was initially interrogated using a multivariate statistical analysis approach (data not reported). This analysis indicated that glucose was the only metabolite capable of differentiating between the various classes, and therefore a targeted analysis of glucose alone was conducted as described below. One sample was missing, and several were haemolysed and not used for data collection. NMR data were collected from $8,11,4$, and 8 samples, for the control 280, 560 and $840 \mu \mathrm{g} / \mathrm{kg}$ feed group, respectively. The signals for the H1 proton of $\alpha$ and $\beta$ glucose are well resolved in the NMR spectrum and thus readily measured for concentration quantification; those for the other glucose protons (which fall in a region 3.20-3.91 ppm) overlap with signals of other metabolites [20] and are not readily quantified. Samples were thawed at room temperature, and $150 \mu \mathrm{L}$ of plasma was added to $\mathrm{D}_{2} \mathrm{O}(600 \mu \mathrm{L}$, with $0.17 \% \mathrm{w} / \mathrm{v}$ 3-(trimethylsilyl)propanoic-2,2,3,3-d4 acid (TSP) (SigmaAldrich, Poole UK). The resulting solution was mixed before centrifugation at $14,000 \mathrm{rpm}$ for 15 minutes. $600 \mu \mathrm{L}$ of the solution was then transferred to a $5 \mathrm{~mm}$ NMR tube (528PPWILMAD, Sigma-Aldrich, Poole, UK). The TSP provided a chemical shift reference $\left(\delta_{\mathrm{H}}=0.00 \mathrm{ppm}\right)$ point and a concentration standard. ${ }^{1} \mathrm{H}$ NMR spectra were acquired at 499.97 MHz on a Varian Unity Inova 500 spectrometer. Spectra were collected using a Carr-Purcell-Meiboom-Gill [RD-90 ${ }_{x}^{\circ}-\left(\tau-180_{y}^{\circ}-\tau\right)_{n}$-collect free induction decay] pulse sequence. The relaxation delay (RD) was 2.0 seconds and the $90^{\circ}$ pulse width was measured for each sample. The spin-spin relaxation delay, $2 n \tau$, of $450 \mathrm{~ms}$ was used for all samples; $\tau$ of $1.5 \mathrm{~ms}$ and $n$ of 150 . The total relaxation delay was three times the $\mathrm{T}_{1}$ measured for TSP. 512 transients were collected into 32,768 data points, with a spectral width of $8,000 \mathrm{~Hz}$. A $1 \mathrm{~Hz}$ line broadening was applied prior to Fourier transformation and baseline correction applied prior to data quantification. The alpha and beta glucose $\mathrm{H} 1$ peaks were integrated using the Advanced Chemistry Development (ACD version 12.01) programme. The integrals were combined for univariate statistical analysis. Univariate analysis was undertaken in SPSS 15.0 (SPSS Inc. Chicago, Illinois, USA) to determine if the data was normally distributed, with parametric or non-parametric testing undertaken following tests of normality. The known concentration of TSP allowed glucose concentrations in the individual samples to be calculated and average concentrations for the different classes of DON exposure.

2.3. Plasma DON Analysis. Plasma samples were available for five, five, four, and six samples for groups 1 to 4, respectively for DON measurements. The amount of DON in plasma was analysed using a modified protocol based on a urinary assay for DON $[8,19]$. Plasma proteins were isolated from $0.5 \mathrm{ml}$ of sample using a 10,000 molecular weight (MW) filter according to manufactures instructions (Millipore, Consett Co, Durham, UK). The protein free plasma was then stored at $-20^{\circ} \mathrm{C}$ whilst the isolated plasma proteins were digested overnight in $1 \mathrm{ml}$ of PBS pH 7.4 containing $0.2 \mathrm{mg}$ Protease Type XIV (Sigma-Aldrich, Poole Dorset, UK) at $37^{\circ} \mathrm{C}$ with gentle shaking. Following protease digestion of the protein pellet, residual enzyme and protein were isolated using 10,000 MW filters, as previously described and the residual protease digest was used to assess the levels of any residual DON and DON glucuronide. The protein free-plasma that was stored and the protease digested were separately spiked with an internal standard (IS) ${ }^{13} \mathrm{C}_{15}$ DON (SigmaAldrich, Poole, Dorset, UK) to a final concentration of $5 \mathrm{ng} / \mathrm{mL}$, prior to overnight treatment with $\beta$-glucuronidase 
as described by Turner [8]. DON residues were then isolated by immunoaffinity and LC-MS as previously described $[8$, 19]. Two spiked controls (PBS containing $5 \mathrm{ng} / \mathrm{mL}$ DON) and a blank control were extracted with the samples, all of which were spiked with the IS. The limit of detection was $0.5 \mathrm{ng} / \mathrm{mL}$. For both sample types DON concentrations are expressed in terms of DON $\mathrm{ng} / \mathrm{mL}$ plasma.

2.4. Statistical Analysis. The mean concentrations of glucose in each animal group were compared using the Student $t$ test (unpaired, two-sided). The trends in plasma glucose, protein-associated DON plasma protein-free DON were assessed using the "nptrend" command in STATA. Linear regression was used to assess the relationship between the DON measures and the glucose concentrations. All data were analysed using STATA v11.

\section{Results}

The mean glucose concentration and 95\% CI at each DON dose are shown in Figure 1. There were no significant differences between the glucose concentrations for the control (5.2 mmolar, 95\% CI: 2.7, 7.6) and the two lower feeding levels of DON $280 \mu \mathrm{g} / \mathrm{Kg}(6.1 \mathrm{mmolar} 95 \%$ CI: 3.7, 8.5) and the $560 \mu \mathrm{g} / \mathrm{Kg}$ (5.3 mmolar, $95 \% \mathrm{CI}$ : 3.7, 5.3), $P>0.6$ for all comparisons. However, the glucose concentration for the $840 \mu \mathrm{g} / \mathrm{kg}$ fed animals (9.4 mmolar, 95\% CI: 6.1, 12.7) was significantly increased compared to the control $(P<0.03)$, and overall there was a modest significant trend to higher plasma glucose with DON dose, $(P=0.04)$.

Overall the levels of DON in protein-free plasma and plasma proteins were low for all samples, range nondetectable to $3.1 \mathrm{ng} / \mathrm{mL}$. DON was not detected in any control protein free-plasma samples, and a modest positive dose response was observed $(P$ for trend $=0.04)$; mean values were $0.4 \mathrm{ng} / \mathrm{mL}$ (SD 0.3), $0.8 \mathrm{ng} / \mathrm{mL}$ (SD 0.5), and $1.0 \mathrm{ng} / \mathrm{mL}$ (SD 0.2) for feeding regimes containing 280, 560 and $840 \mu \mathrm{g}$ DON $/ \mathrm{kg}$ feed respectively, see Figure 2. For the plasma protein fraction the control samples contained measurable DON equivalent to $0.8 \mathrm{ng} / \mathrm{mL}$ (SD 0.3) plasma, whilst a nonsignificant positive trend was observed with increasing dose of $\mathrm{DON}(P=0.08)$; mean values (SD) were $1.0 \mathrm{ng} / \mathrm{mL}(0.2)$, $1.8 \mathrm{ng} / \mathrm{mL}(0.9)$ and $2.1 \mathrm{ng} / \mathrm{mL}(0.7)$, respectively.

Where paired data were available there was a nonsignificant association between the glucose concentration and both protein-free plasma DON concentration and protein-associated DON, $\left(P<0.09, r^{2}=0.04\right.$ and $P<0.06$, $r^{2}=0.06$, resp., data not shown). The significance of this observation remains limited due to low numbers.

\section{Discussion}

The NMR study revealed that swine in the highest intake group had significantly higher levels of serum glucose compared to controls, and overall there was a trend to higher glucose levels across the study $(P=0.04)$. In all groups of animals the mean glucose concentration was higher than that reported previously, which most likely reflects a systemic

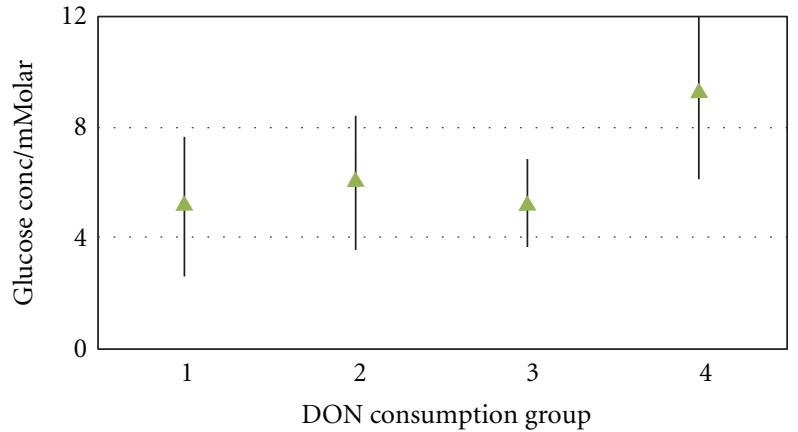

FIgURE 1: Glucose concentration as a function of dietary DON. Mean and 95\%CI Group 1:control, Group 2: $240 \mu \mathrm{g} / \mathrm{kg}$, Group 3: $560 \mu \mathrm{g} / \mathrm{Kg}$, Group 4: $840 \mu \mathrm{g} / \mathrm{Kg}$.

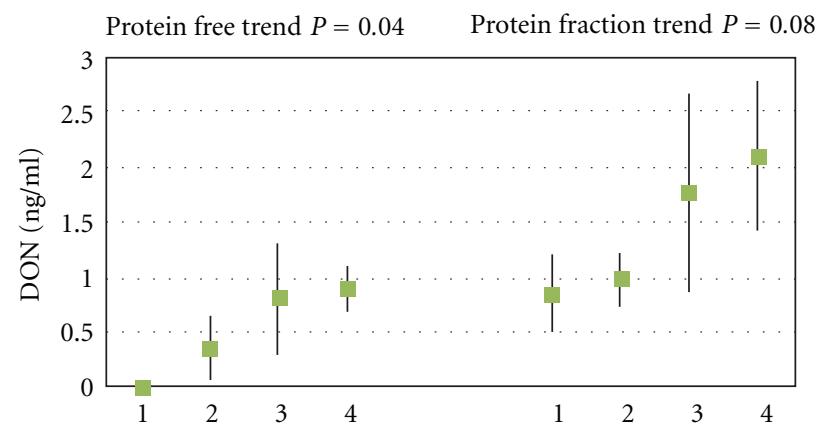

FIGURE 2: DON concentration in protein-free plasma and protein fraction in relation to dietary DON intake.

effect of the NMR-based approach. The TSP level measured is likely to be lower than the concentration incorporated with the sample. This is because there is a loose association between TSP and albumin, and any TSP interacting with albumin will not contribute to the TSP signal detected using the data collection procedure adopted here. This would therefore have the effect of producing a higher apparent glucose concentration, though only slightly. The difference would be the same across the sample collection as TSP levels were found to be invariant. Given that DON has been reported to inhibit glucose transporters in vitro using isolated jejunal sections from hens $[15,16]$ our observation was slightly surprising. Ex vivo investigations of the effect of DON on glucose transporters from jejunal sections of swine revealed a nonsignificant modest increase in glucose transport rate rather than inhibition, compared to controls [21]. However these two sets of experiments were not equivalent. Apart from the species difference, the former $[15,16]$ investigated the effects of DON directly on isolated jejunal tissue, whilst the latter [21] involved animals dosed with DON prior to jejunal isolation. This latter whole animal study provides some support for our observed increase in plasma glucose in swine. A separate study in rats also revealed disturbed insulin and glucagon homeostasis following short term (three days) DON treatment [22]. These authors suggest a direct effect of DON on the pancreas, and their observations include a significantly higher serum glucose 
concentration in the DON treated compared to control animals. It is of course plausible, though unproven, that this effect on the pancreas may reflect inhibition of pancreatic, $\beta$ cell glucose transporters, which are predominantly GLUT2. As previously mentioned higher doses of DON in swine ( $>2 \mathrm{mg} / \mathrm{kg}$ in feed) have also been associated with increased plasma glucose concentrations [22]. Whilst the mechanism for our observations awaits further study, it is important to indicate that much of the literature data has used doses of DON that may not be relevant to typical human exposures. Our survey in swine does include lower, and perhaps more, relevant doses.

Meky et al. [23] conducted a series of preliminary investigations using a rat model, and a set of human urines in a pilot study that highlighted urinary DON as a potential exposure biomarker. This putative urinary biomarker was subsequently validated $[8,19]$. The original rat model also highlighted that about $10 \%$ of the plasma DON was attached to or associated with plasma proteins [23]. Due to the relatively rapid clearance of DON from the peripheral circulation, plasma levels of DON may not be ideal as an exposure biomarker, at typical human levels. However, if serum proteins serve as a "carrier" of DON, then these may provide a less transient matrix for biomeasurements and biomonitoring. In this study two types of biomeasures of DON were investigated; firstly DON was measured in the protein-free fraction (for proteins $>10,000 \mathrm{MW}$ ) of plasma, and secondly, in the protein fraction. A positive dose response was observed for the plasma fraction $(P=0.04)$ and for the protein fraction $(P=0.08)$, though of borderline significance in the latter. Overall DON levels were low in all samples and were all within approximately six times the limit of quantitation. The amount of DON in protein free sera or plasma is not likely to provide a useful biomarker of exposure for humans due to its variability in proximity to DON ingestion. The temporal nature of protein associated fraction remains to be demonstrated, but perhaps serves as a more stable and slightly longer-term measure of average DON intake. The data presented here offer modest support, though indicate that analytical methods need to be further developed and greater sensitivity will likely be required. The relationship between DON measures and the glucose concentrations was at best modest and nonsignificant. A number of caveats should be highlighted, not least of which was the small sample size for these observations; the modest association between intake and the putative DON biomeasures, and a lack of data on the stability of DON in cryopreserved plasma samples.

In conclusion, this study revealed a modest dose response effect between dietary DON and plasma glucose measured by NMR. It is plausible that the homeostatic control of insulin could be disturbed by DON, and thus glucose uptake from the peripheral circulation may be restricted. Given the limited number of animals and that the effect only occurred at the highest dose raises some caution as to the relevance to typical human exposures. Plasma DON was also detected and DON was found to be associated with the plasma protein fraction. The nature of the association remains unknown, but is not likely to be a covalent interaction, as DON itself rather than DON residues was isolated and measured by LC-MS. Turner and colleagues have developed and validated a urinary biomarker for DON exposure. A longer-term biomeasure would be of great value to support epidemiological investigations, and the preliminary data reported here should support further development of this approach.

\section{Abbreviations}

DON: Deoxynivalenol

Ig: Immunoglobulin

NMR: Nuclear magnetic resonance

TSP: 3-(trimethylsilyl) propanoic acid sodium salt.

\section{Conflict of Interests}

The authors declare that there are no conflict of interests.

\section{Acknowledgments}

The thank the White Rose Doctoral Training Centre, UK (R.P.H). The financial support of the European Union for the Project "MycoRed" (FP7-KBBE-2007-2A) is greatly acknowledged. The authors are solely responsible for the work described in this paper, and their opinions are not necessarily those of the European Union. They thank Kay White (University of Leeds) for LC-MS support.

\section{References}

[1] CAST. Council for Agricultural Science and Technology, "Potential economic costs of mycotoxins in the United States," in Mycotoxins: Risks in Plant, Animal and Human Systems, Task Force Report No. 139, pp. 136-142, Ames, Iowa, USA, 2003.

[2] SCOOP, In Collection of occurrence data of Fusarium toxins in food and assessment of dietary intake by the population of EU member states. SCOOP Task 3. 2. 10 Final report, 2003, http://ec.europa.eu/food/fs/scoop/task3210.pdf.

[3] L. S. Jackson and L. B. Bullerman, "Effect of processing on Fusarium mycotoxins," Advances in Experimental Medicine and Biology, vol. 459, pp. 243-261, 1999.

[4] Y. Sugita-Konsihi, T. Tanaka, S. Tabata et al., "Validation of an HPLC analytical method coupled to a multifunctional clean-up column for the determination of deoxynivalenol," Mycopathologia, vol. 161, no. 4, pp. 239-243, 2006.

[5] SCF, Opinion of the Scientific Committee on Food on Fusarium toxins. Part 6: Group evaluation of T-2 toxin, HT-2 toxin, nivalenol and deoxynivalenol: SCF/CS/CNTM/MYC/27 Final, 2002, http://ec.europa.eu/food/fs/sc/scf/out123_en.pdf.

[6] J. J. Pestka and A. T. Smolinski, "Deoxynivalenol: toxicology and potential effects on humans," Journal of Toxicology and Environmental Health B, vol. 8, no. 1, pp. 39-69, 2005.

[7] P. C. Turner, R. P. Hopton, Y. Lecluse, K. L. M. White, J. Fisher, and P. Lebailly, "Determinants of urinary deoxynivalenol and de-epoxy deoxynivalenol in male farmers from normandy, France," Journal of Agricultural and Food Chemistry, vol. 58, no. 8, pp. 5206-5212, 2010.

[8] P. C. Turner, "Deoxynivalenol and nivalenol occurrence and exposure assessment," World Mycotoxin Journal, vol. 3, no. 4, pp. 315-321, 2010. 
[9] G. Sundstøl Eriksen and H. Pettersson, "Lack of deepoxidation of type B trichothecenes in incubates with human faeces," Food Additives \& Contaminants, vol. 20, no. 6, pp. 579582, 2003.

[10] S. J. Hepworth, L. J. Hardie, L. K. Fraser et al., "Deoxynivalenol exposure assessment in a cohort of pregnant women from Bradford, UK," Food Additives \& Contaminants A, vol. 29, no. 2, pp. 269-276, 2012.

[11] P. C. Turner, R. P. Hopton, K. L. M. White, J. Fisher, J. E. Cade, and C. P. Wild, "Assessment of deoxynivalenol metabolite profiles in UK adults," Food and Chemical Toxicology, vol. 49, no. 1, pp. 132-135, 2011.

[12] R. P. Hopton, E. Turner, V. J. Burley, P. C. Turner, and J. Fisher, "Urine metabolite analysis as a function of deoxynivalenol exposure: an NMR-based metabolomics investigation," Food Additives \& Contaminants A, vol. 27, no. 2, pp. 255-261, 2010.

[13] A. Nogueira da Costa, R. S. Mijal, J. N. Keen, J. B. C. Findlay, and C. P. Wild, "Proteomic analysis of the effects of the immunomodulatory mycotoxin deoxynivalenol," Proteomics, vol. 11, no. 10, pp. 1903-1914, 2011.

[14] A. Nogueira da Costa, J. N. Keen, C. P. Wild, and J. B. C. Findlay, "An analysis of the phosphoproteome of immune cell lines exposed to the immunomodulatory mycotoxin deoxynivalenol," Biochimica et Biophysica Acta, vol. 1814, no. 7, pp. 850-857, 2011.

[15] W. A. Awad, J. R. Aschenbach, F. M. C. S. Setyabudi, E. Razzazi-Fazeli, J. Böhm, and J. Zentek, "In vitro effects of deoxynivalenol on small intestinal D-glucose uptake and absorption of deoxynivalenol across the isolated jejunal epithelium of laying hens," Poultry Science, vol. 86, no. 1, pp. 15-20, 2007.

[16] W. A. Awad, E. Razzazi-Fazeli, J. Böhm, and J. Zentek, "Effects of B-trichothecenes on luminal glucose transport across the isolated jejunal epithelium of broiler chickens," Journal of Animal Physiology and Animal Nutrition, vol. 92, no. 3, pp. 225-230, 2008.

[17] P. Pinton, F. Accensi, E. Beauchamp et al., "Ingestion of deoxynivalenol (DON) contaminated feed alters the pig vaccinal immune responses," Toxicology Letters, vol. 177, no. 3, pp. 215-222, 2008.

[18] F. Accensi, P. Pinton, P. Callu et al., "Ingestion of low doses of deoxynivalenol does not affect hematological, biochemical, or immune responses of piglets," Journal of Animal Science, vol. 84, no. 7, pp. 1935-1942, 2006.

[19] P. C. Turner, V. J. Burley, J. A. Rothwell, K. L. M. White, J. E. Cade, and C. P. Wild, "Dietary wheat reduction decreases the level of urinary deoxynivalenol in UK adults," Journal of Exposure Science and Environmental Epidemiology, vol. 18, no. 4, pp. 392-399, 2008.

[20] J. K. Nicholson, P. J. D. Foxall, M. Spraul, R. D. Farrant, and J. C. Lindon, "750 MHz 1H and 1H-13C NMR spectroscopy of human blood plasma," Analytical Chemistry, vol. 67, no. 5, pp. 793-811, 1995.

[21] K. Zerull, G. Breves, B. Schroder, T. Goyarts, and S. Danicke, "The influence of the mycotoxin deoxynivalenol on jejunal glucose transport in pigs," Mycotoxin Research, vol. 21, no. 4, pp. 251-257, 2005.

[22] K. Szkudelska, T. Szkudelski, and L. Nogowski, "Short-time deoxynivalenol treatment induces metabolic disturbances in the rat," Toxicology Letters, vol. 136, no. 1, pp. 25-31, 2002.

[23] F. A. Meky, P. C. Turner, A. E. Ashcroft et al., "Development of a urinary biomarker of human exposure to deoxynivalenol," Food and Chemical Toxicology, vol. 41, no. 2, pp. 265-273, 2003. 


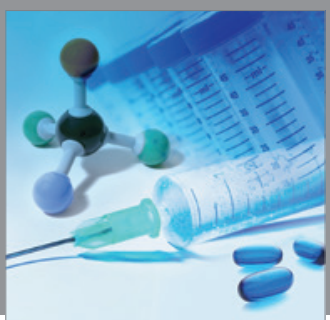

International Journal of

Medicinal Chemistry

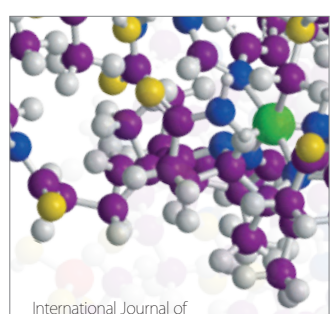

Carbohydrate Chemistry

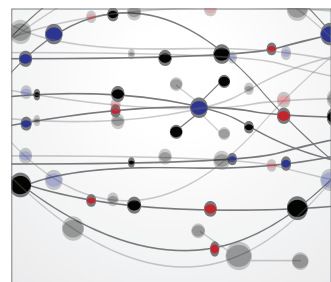

The Scientific World Journal
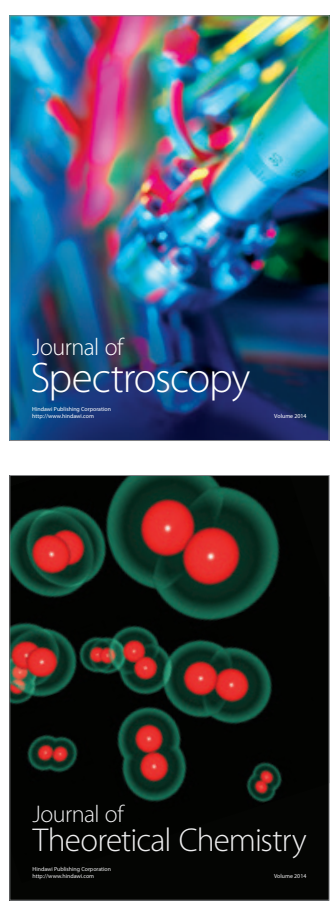
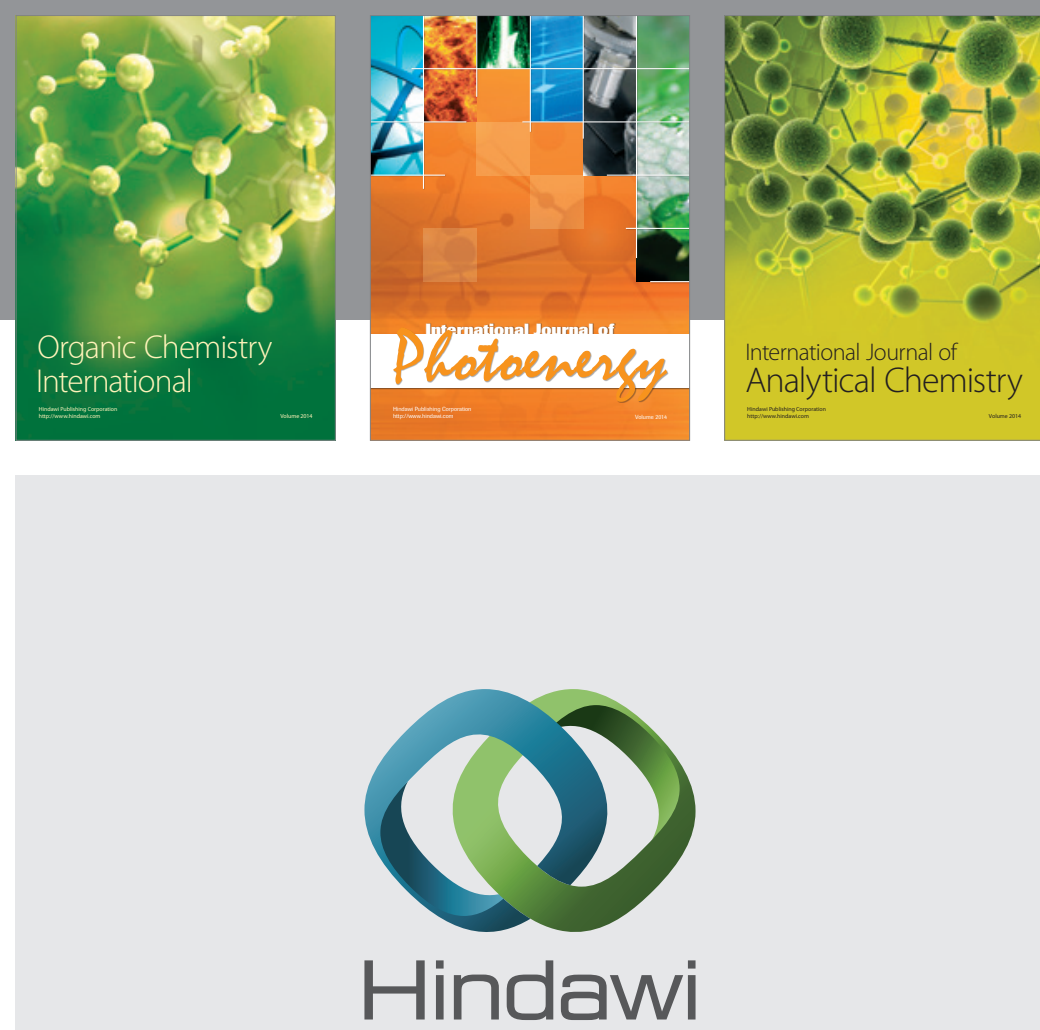

Submit your manuscripts at

http://www.hindawi.com
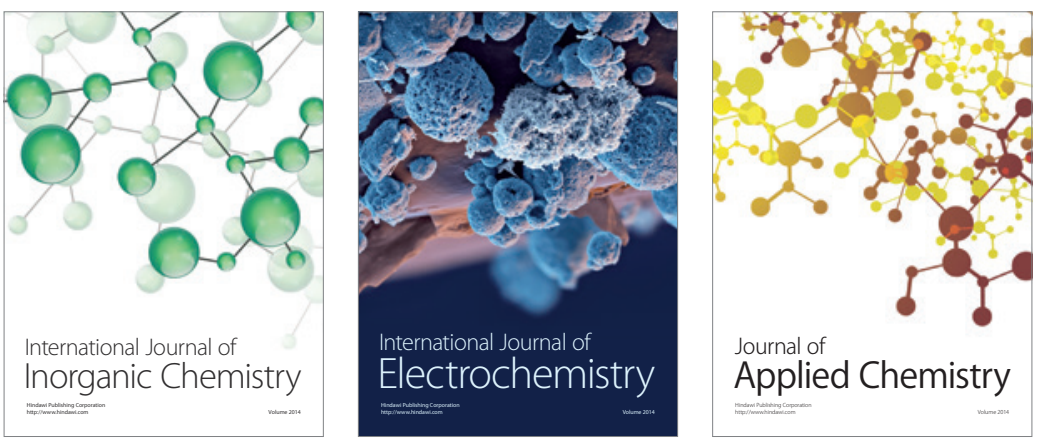

Journal of

Applied Chemistry
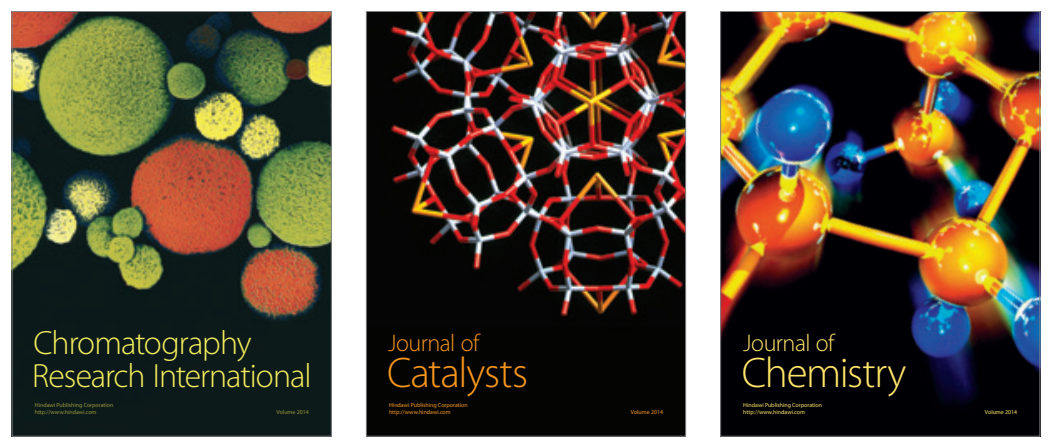
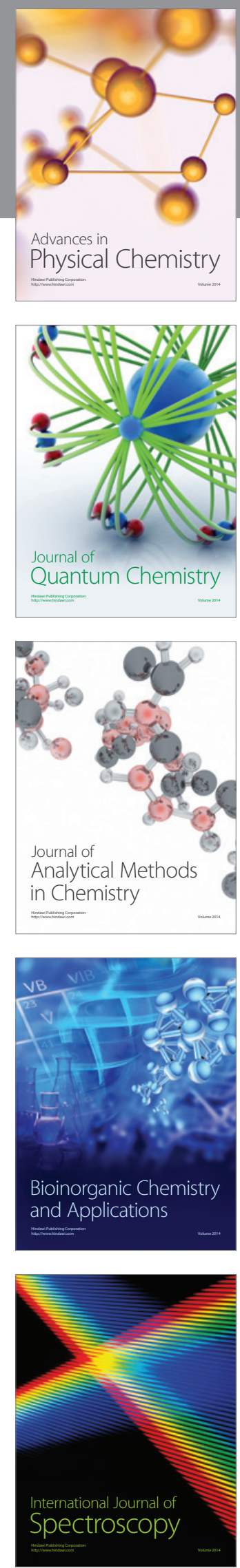